\title{
The Effect of Loop Diuretics on Sarcopenia and Long-Term Prognosis in Patients with Refractory Hepatic Ascites Treated with Tolvaptan
}

\author{
Masaaki Shimada*, Hiroaki Iwase, Noboru Hirashima, Masashi Saito, Hisashi Kondo, \\ Noboru Urata, Satoshi Unita, Takashi Kondo, Daiki Tanaka, Takuya Tsunekawa \\ Department of Gastroenterology, National Hospital Organization, Nagoya Medical Center, Nagoya, Japan \\ Email: *shimada@nnh.hosp.go.jp
}

How to cite this paper: Shimada, M., Iwase, H., Hirashima, N., Saito, M., Kondo, H., Urata, N., Unita, S., Kondo, T., Tanaka, D. and Tsunekawa, T. (2018) The Effect of Loop Diuretics on Sarcopenia and Long-Term Prognosis in Patients with Refractory Hepatic Ascites Treated with Tolvaptan. Open Journal of Gastroenterology, 8, 201-208. https://doi.org/10.4236/ojgas.2018.86022

Received: May 7, 2018

Accepted: June 12, 2018

Published: June 15, 2018

Copyright $\odot 2018$ by authors and Scientific Research Publishing Inc. This work is licensed under the Creative Commons Attribution International License (CC BY 4.0).

http://creativecommons.org/licenses/by/4.0/

\begin{abstract}
We investigated sarcopenia, focusing on the dose of loop diuretics used in 70 patients with refractory hepatic ascites treated with tolvaptan. Bloating improved in $68.5 \%$ of patients, as determined using the Japanese version of the Support Team Assessment Schedule. The psoas muscle index (PMI) was used to define sarcopenia. A statistically significant difference was observed in the PMI between patients receiving low-dose $\left(3.6 \pm 1.2 \mathrm{~cm}^{2} / \mathrm{m}^{2}\right)$ and high-dose furosemide $\left(3.1 \pm 1.2 \mathrm{~cm}^{2} / \mathrm{m}^{2}\right)$ before tolvaptan treatment $(P=0.048)$. The PMI increased from $3.2 \pm 1.1 \mathrm{~cm}^{2} / \mathrm{m}^{2}$ to $3.5 \pm 1.3 \mathrm{~cm}^{2} / \mathrm{m}^{2}(P=0.002)$ in responders, but decreased from $3.4 \pm 1.2 \mathrm{~cm}^{2} / \mathrm{m}^{2}$ to $3.0 \pm 1.0 \mathrm{~cm}^{2} / \mathrm{m}^{2}(P=0.106)$ in non-responders before and after tolvaptan treatment, respectively. The long-term prognosis improved in responders compared with non-responders (mean survival time: 646 days vs. 228 days, $P<0.001$ ). Early introduction of tolvaptan treatment is necessary to prevent the progression of sarcopenia.
\end{abstract}

\section{Keywords}

Tolvaptan, Hepatic Ascites, Sarcopenia, Loop Diuretics, Long-Term Prognosis

\section{Introduction}

Refractory hepatic ascites that cannot be controlled with conventional therapy by using loop diuretics and spironolactone greatly reduces a patient's general status and quality of life [1]. Hypoalbuminemia and hyponatremia are frequently observed in these cases, and the long-term prognosis worsens [2]. 
Tolvaptan was recently approved in Japan for refractory hepatic ascites that demonstrates an insufficient response to loop diuretics. Tolvaptan is a new type of water diuresis medication, i.e., a solute-free water excretion mediator that promotes a diuretic effect by inhibiting reabsorption of water, with vasopressin $\mathrm{V}_{2}$-receptor antagonism in the kidney collecting tubules [3]. Bloatedness was significantly improved in $62.5 \%$ in the tolvaptan group and $37.3 \%$ in the placebo group in a Japanese phase III clinical trial. Therefore, tolvaptan exerts an effect on refractory hepatic ascites [2].

It was reported that $63 \%$ of patients with hepatic cirrhosis have malnutrition [4], and that cases with sarcopenia have a poor prognosis; therefore, sarcopenia assessment criteria specific for hepatic disorders were proposed in 2016 by the Japan Society of Hepatology [5].

$\mathrm{Na}-\mathrm{K}-2 \mathrm{Cl}$ cotransporter protein, the target of loop diuretics in a mouse model, is a control factor for myoblastic differentiation and skeletal muscle hypertrophy [6]. In other words, muscular enlargement occurred with exercise in a normal mouse, but muscular hypertrophy could not develop in a mouse receiving a loop diuretic. These results suggested that the frequency of sarcopenia may increase in patients receiving a high-dose loop diuretic. However, whether sarcopenia is associated with the dose of loop diuretics in patients with refractory hepatic ascites treated with tolvaptan remains unclear.

Therefore, we evaluated the association between loop diuretic dose and sarcopenia in refractory hepatic ascites treated with tolvaptan.

\section{Materials and Methods}

We retrospectively studied data for a total of 70 consecutive patients with refractory hepatic ascites who did not respond adequately to conventional diuretics and were treated between April 2011 and April 2017 with tolvaptan. Low-dose use of a loop diuretic (furosemide) was defined as $20 \mathrm{mg} /$ day or less, and high-dose use as over $20 \mathrm{mg} /$ day. Bloatedness from hepatic ascites was scored on a scale of 0 to 4 using the Japanese version of the Support Team Assessment Schedule (STAS-J) [7] before and after 3 weeks of tolvaptan treatment. The STAS-J is scored from 0 to $4-0$ : no symptoms; 1 : one to several, occasional or intermittent, concurrent symptoms that do not interfere with activities of daily living and do not require treatment; 2 : moderate symptoms on some days, which may affect the ability to perform activities of daily living; 3: frequent severe symptoms that significantly affect the ability to perform activities of daily living; 4: continuous, intense or intolerable symptoms, preventing ability to perform activities of daily living. A patient was defined as a responder when the score decreased by 1 point or more and as a nonresponder when the score did not change or increase by 1 point or more after tolvaptan administration. The dose of loop diuretic before tolvaptan treatment, the changes in caloric intake during hospitalization, and the psoas major muscle area measured with computed tomography (CT) at the level of the third lumbar vertebra (L3) were eva- 
luated. The psoas muscle index (PMI) was obtained by tracing an outline of the bilateral psoas major using an image unification system (SYNAPSE SCOPE V2 4.1; Fujifilm Corporation, Tokyo, Japan) at the level of L3 on an abdominal axial CT image and the total area was divided by the square of body height. The cut-off value was $6.36 \mathrm{~cm}^{2} / \mathrm{m}^{2}$ for men and $3.92 \mathrm{~cm}^{2} / \mathrm{m}^{2}$ for women, as previously reported [5], and sarcopenia was defined according to the PMI. We evaluated long-term patient prognosis. Overall survival was defined by the time period from the date of patient registration until death, or the final date when survival was confirmed.

\section{Statistical Analysis}

All data were expressed as the mean \pm standard deviation (SD). Differences were analyzed using the Wilcoxon signed-rank test, two-sample t-test, and chi-square test. The long-term prognosis was estimated using the Kaplan-Meier method, and differences were determined using the log-rank test. $P$-values $<0.05$ were considered statistically significant.

This study was approved by the ethics review board of the Nagoya Medical Center.

\section{Results}

\subsection{Baseline Patient Characteristics (Table 1)}

The study included 28 men and 42 women, with an average age of $69.1 \pm 13.0$ years. The etiology of chronic liver disease was as follows: alcoholic, 17 cases;

Table 1. Baseline patient characteristics.

$\begin{array}{cc}\text { Age (years) } & 69.1 \pm 13.0 \\ \text { Sex (male/female) } & 28 / 42 \\ \text { Body weight (kg) } & 58.6 \pm 13.5 \\ \text { Etiology of chronic liver disease } & \\ \text { Alcoholic/Hepatitis B/Hepatitis C/PBC/AIH/Others } & 17 / 3 / 26 / 9 / 5 / 10 \\ \text { HCC } & 21 \\ \text { Child-Pugh classification (B/C) } & 27 / 43 \\ \text { Child-Pugh score } & 10.1 \pm 1.6 \\ \text { PMI (cm }{ }^{2} / \mathrm{m}^{2} \text { ) } & 3.3 \pm 1.2 \\ \text { STAS-J score } & 3.3 \pm 0.6 \\ \text { Caloric intake (kcal) } & 972 \pm 407 \\ \text { Furosemide (mg/day) } & 38.6 \pm 22.0 \\ \text { Tolvaptan (mg/day) } & 8.2 \pm 2.6 \\ \text { Data are expressed as number or mean } \pm \text { SD }\end{array}$

PBC: primary biliary cirrhosis, AIH: autoimmune hepatitis, HCC: hepatocellular carcinoma, PMI: L3 level psoas muscle index, STAS-J: Japanese version Support Team Assessment Schedule. 
hepatitis type B, 3; hepatitis type C, 26; primary biliary cirrhosis, 9; autoimmune hepatitis, 5; and others, 10. Twenty-one patients had hepatocellular carcinoma. According to the Child-Pugh classification [8], 27 were in class B and 43 in class C; the mean Child-Pugh score was 10.1 \pm 1.6 .

\subsection{Efficacy of Tolvaptan (Table 2)}

The rate of low-dose loop diuretic (furosemide, $\leq 20 \mathrm{mg} /$ day) use was $41.4 \%$ and that of high-dose ( $>20 \mathrm{mg} /$ day) use was $58.6 \%$ before tolvaptan treatment. The mean STAS-J score significantly decreased from $3.3 \pm 0.6$ to $2.1 \pm 1.0(P<0.001)$ after tolvaptan administration and bloatedness improved in 48 patients $(68.5 \%)$. Patient body weight significantly decreased from $56.0 \pm 12.2 \mathrm{~kg}$ to $52.8 \pm 11.8 \mathrm{~kg}$ $(P<0.001)$, and the caloric intake increased from $972 \pm 407 \mathrm{kcal}$ to $1141 \pm 410$ $\mathrm{kcal}(P=0.001)$ in 3 weeks after administration of tolvaptan.

\subsection{The Frequency of Sarcopenia}

The rate of sarcopenia was $90 \%$ (men, $100 \%$; women, $83.3 \%$ ). A statistically significant difference was observed in PMI between low-dose $\left(3.6 \pm 1.2 \mathrm{~cm}^{2} / \mathrm{m}^{2}\right)$ and high-dose use of furosemide $\left(3.1 \pm 1.2 \mathrm{~cm}^{2} / \mathrm{m}^{2}\right)$ before tolvaptan treatment $(P=0.048)$ (Figure 1$)$. However, there was no significant difference between low-dose $\left(3.7 \pm 1.1 \mathrm{~cm}^{2} / \mathrm{m}^{2}\right)$ and high-dose use of furosemide $\left(3.2 \pm 1.3 \mathrm{~cm}^{2} / \mathrm{m}^{2}\right)$

Table 2. Effect of treatment.

\begin{tabular}{cccc}
\hline & Before treatment & 3 weeks & $P$-value $^{\dagger}$ \\
\hline STAS-J score & $3.3 \pm 0.6$ & $2.1 \pm 1.0$ & $<0.001$ \\
Body weight $(\mathrm{kg})$ & $56.0 \pm 12.2$ & $52.8 \pm 11.8$ & $<0.001$ \\
Caloric intake $(\mathrm{kcal})$ & $972 \pm 407$ & $1,141 \pm 410$ & 0.001 \\
\hline
\end{tabular}

Data are expressed as mean \pm SD. ${ }^{\dagger}$ Wilcoxon signed-rank test (relative to baseline). STAS-J: Japanese version Support Team Assessment Schedule.

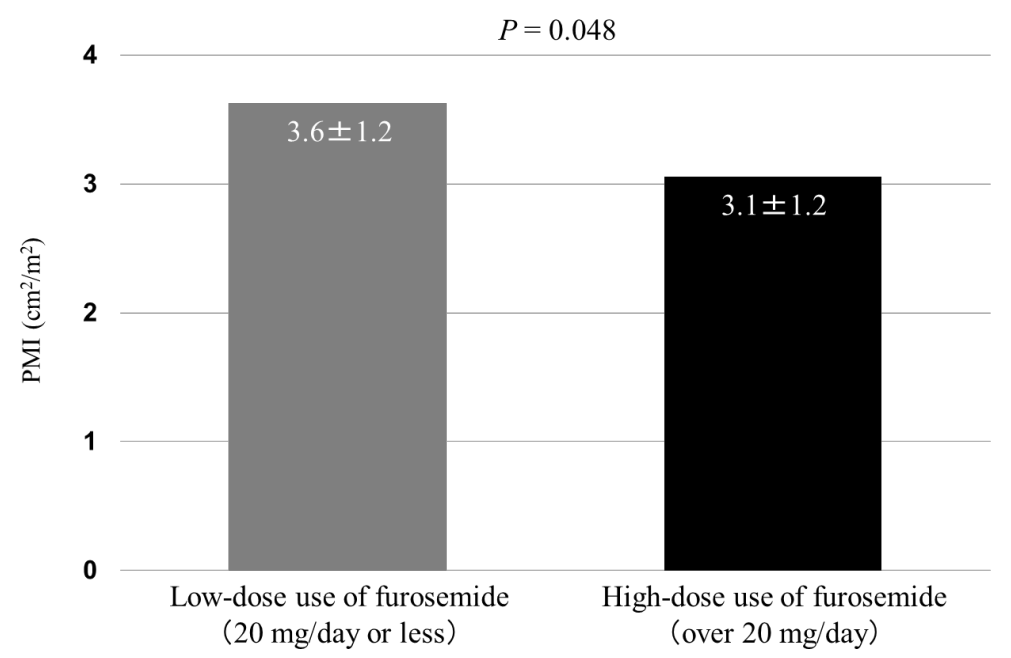

Figure 1. The association between loop diuretic dose and PMI. 
after tolvaptan treatment $(P=0.165)$. There were 22 responders $(75.9 \%)$ and 7 non-responders $(24.1 \%)$ in the low-dose furosemide group, and 26 responders (63.4\%) and 15 non-responders (36.6\%) in the high-dose group ( $P=0.269)$. The PMI increased from $3.2 \pm 1.1$ to $3.5 \pm 1.3 \mathrm{~cm}^{2} / \mathrm{m}^{2}(P=0.002)$ in responders, but decreased from $3.4 \pm 1.2$ to $3.0 \pm 1.0 \mathrm{~cm}^{2} / \mathrm{m}^{2}(P=0.106)$ in non-responders before and after tolvaptan treatment, respectively (observation period; $124 \pm 114$ days) (Table 3).

\subsection{Long-Term Prognosis}

The mean survival time was 533 days in all patients (Figure 2(a)), with 228 days in non-responders and 646 days in responders $(P<0.001)$. The long-term prognosis improved in responders compared with non-responders (Figure 2(b)).

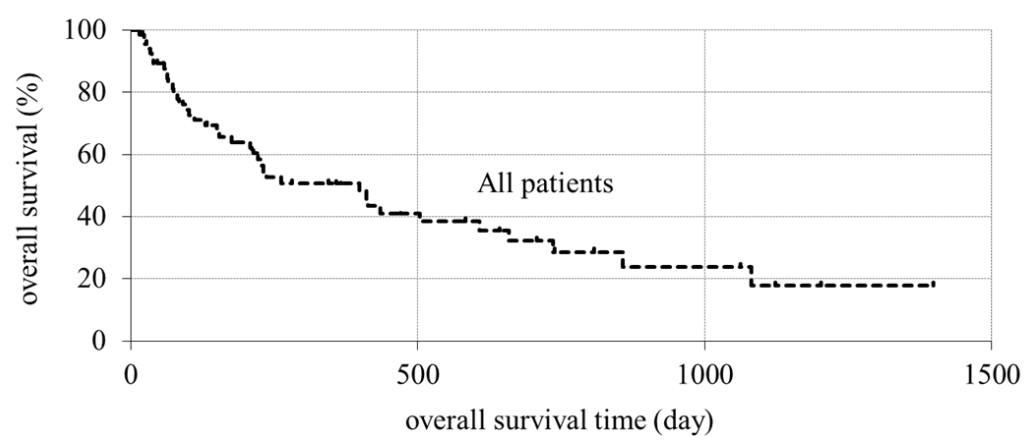

(a)

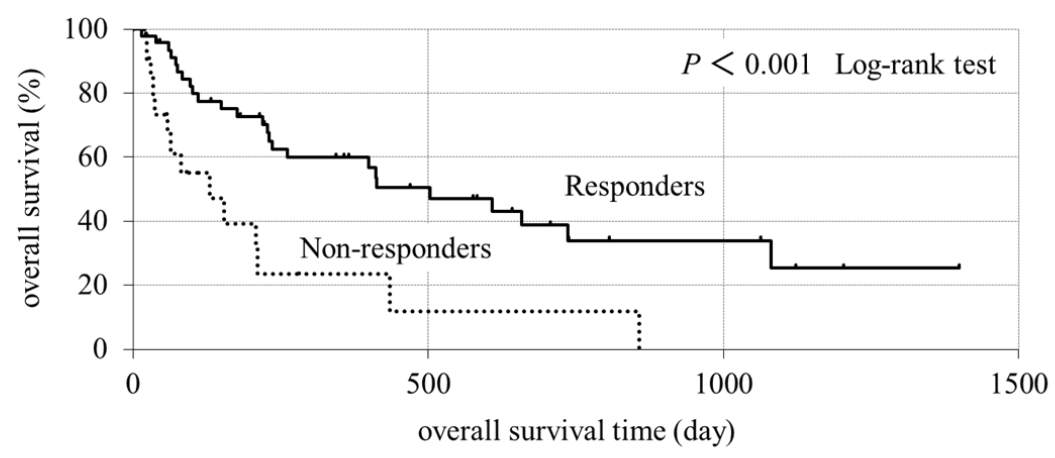

(b)

Figure 2. Long-term prognosis (a) all patients; (b) comparison between responders and non-responders).

Table 3. Change in PMI according to the effect of tolvaptan.

\begin{tabular}{cccc}
\hline \multicolumn{2}{c}{ PMI $\left(\mathrm{cm}^{2} / \mathrm{m}^{2}\right)$} & $P$-value \\
\hline Before treatment & After treatment & \\
\hline Responders & $3.2 \pm 1.1$ & $3.5 \pm 1.3$ & 0.002 \\
Non-responders & $3.4 \pm 1.2$ & $3.0 \pm 1.0$ & 0.106 \\
Data are expressed as mean \pm SD & & \\
\hline
\end{tabular}

${ }^{\dagger}$ Wilcoxon signed-rank test (relative to baseline). 


\section{Discussion}

Sarcopenia was defined by the European Working Group on Sarcopenia in Older People as a syndrome characterized by a progressive and systemic decrease in muscle mass and strength [9]. Sarcopenia is associated with malignancy, renal failure, heart failure, and aging, and leads to reduction in quality of life and an increased risk of death [10] [11]. The mechanism of sarcopenia is complicated, and is not fully understood, but patient prognosis has improved with management [12].

We were able to control refractory hepatic ascites with tolvaptan and improve nutrition using the Controlling Nutritional Status method [13]. Patients with refractory hepatic ascites had a moderate to high degree of malnutrition in our study [14]. It was reported that appetite improved during treatment with tolvap$\tan$ compared with placebo (38.9\% vs. $16.7 \%, P=0.064)$ in a Japanese phase III clinical trial of patients with hepatic edema [2]. In this study, bloatedness improved in $68.5 \%$ of patients with refractory hepatic ascites after tolvaptan treatment based on the STAS-J score, pain was relieved, and nutritional status improved, as shown by caloric intake.

Skeletal muscle mass is evaluated using arm circumference, dual-energy X-ray absorptiometry, bioelectrical impedance analysis, CT, or magnetic resonance imaging. Sarcopenia assessment criteria were recommended by the Japan Society of Hepatology in 2016 [5]. We examined abdominal CT images before tolvaptan induction and determined that PMI decreased in many cases. Therefore, we presumed that patients with refractory hepatic ascites had sarcopenia.

The prognosis of patients diagnosed with liver cirrhosis and ascites is poor, with $62 \%$ deceased at 6 months, $56 \%$ at 12 months, and $49 \%$ at 24 months [13]. All patients in this study were considered to have end-stage liver cirrhosis that would not respond to conventional diuretics, and their prognosis was extremely poor. If the dose of loop diuretic was high, the frequency of sarcopenia increased in patients with refractory hepatic ascites treated with tolvaptan. Furthermore, the frequency of sarcopenia increased in non-responders, but decreased in patients on a low dose of loop diuretic and in responders treated with tolvaptan. Early introduction of tolvaptan treatment prevented exacerbation of sarcopenia, with improvement in bloatedness and increased caloric intake in this study, and is thought to be extremely important in improving patient prognosis.

This study had some limitations. First, this was a retrospective study in a single institution. Second, we did not study muscle strength, which is important for the evaluation of sarcopenia. Third, we did not study other mechanisms underlying sarcopenia. A larger, multicenter prospective study should be performed to confirm our findings.

\section{Conclusion}

The frequency of sarcopenia in refractory hepatic ascites treated with tolvaptan 
was high. Improvement in sarcopenia was found in responders, and improvement in long-term prognosis was expected. Early introduction of tolvaptan treatment is necessary because sarcopenia is increased in patients treated with high-dose furosemide.

\section{References}

[1] Llach, J., Gines, P., Arroyo, V., et al. (1988) Prognostic Value of Arterial Pressure, Endogenous Vasoactive Systems, and Renal Function in Cirrhotic Patients Admitted to the Hospital for the Treatment of Ascites. Gastroenterology, 94, 482-487. https://doi.org/10.1016/0016-5085(88)90441-6

[2] Sakaida, I., Kawazoe, S., Kajimura, K., et al. (2014) Tolvaptan for Improvement of Hepatic Edema: A Phase 3, Multicenter, Randomized, Double-Blind, Placebo-Controlled Trial. Hepatology Research, 44, 73-82. https://doi.org/10.1111/hepr.12098

[3] Zmily, H.D., Daifallah, S. and Ghali, J.K. (2011) Tolvaptan, Hyponatremia, and Heart Failure. International Journal of Nephrology and Renovascular Disease, 4, 57-71.

[4] Larramona, G.L., Lucendo, A.J. and Tenias, J.M. (2015) Association between Nutritional Screening via the Controlling Nutritional Status Index and Bone Mineral Density in Chronic Liver Disease of Various Etiologies. Hepatology Research, 45, 618-628. https://doi.org/10.1111/hepr.12395

[5] Nishikawa, H., Shiraki, M., Hiramatsu, A., et al. (2016) Japan Society of Hepatology Guidelines for Sarcopenia in Liver Disease (1st Edition): Recommendation from the Working Group for Creation of Sarcopenia Assessment Criteria. Hepatology Research, 46, 951-963. https://doi.org/10.1111/hepr.12774

[6] Mandai, S., Furukawa, S., Kodaka, M., et al. (2017) Loop Diuretics Affect Skeletal Myoblast Differentiation and Exercise-Induced Muscle Hypertrophy. Scientific Reports, 7, Article Number: 46369. https://doi.org/10.1038/srep46369

[7] STAS Working Group. (2007) STAS-J (The Japanese Version Support Team Assessment Schedule) Scoring Manual. For Clinical Audit in the Palliative Care. Japan Hospice/Palliative Care Foundation, 3rd Edition, Koubunsya, Chiba, 1-93.

[8] Pugh, R.N.H., Murray-Lyon, I.M., Dawson, J.L., et al. (1973) Transection of the Oesophagus for Bleeding Oesophageal Varices. The British Journal of Surgery, 60, 646-649. https://doi.org/10.1002/bjs.1800600817

[9] Cruz-Jentoft, A.J., Baeyens, J.P., Bauer, J.M., et al. (2010) Sarcopenia: European Consensus on Definition and Diagnosis: Report of the European Working Group on Sarcopenia in Older People. Age and Ageing, 39, 412-423. https://doi.org/10.1093/ageing/afq034

[10] Wilson, J.R., Mancini, D.M. and Dunkman, W.B. (1993) Exertional Fatigue Due to Skeletal Muscle Dysfunction in Patients with Heart Failure. Circulation, 87, 470-475. https://doi.org/10.1161/01.CIR.87.2.470

[11] Sharma, D., Hawkins, M. and Abramowitz, M.K. (2014) Association of Sarcopenia with eGFR and Misclassification of Obesity in Adults with CKD in the United States. Clinical Journal of the American Society of Nephrology, 9, 2079-2088. https://doi.org/10.2215/CJN.02140214

[12] Zhou, X., Wang, J.L., Lu, J., et al. (2010) Reversal of Cancer Cachexia and Muscle Wasting by ActRIIB Antagonism Leads to Prolonged Survival. Cell, 142, 531-543. https://doi.org/10.1016/j.cell.2010.07.011 
[13] Ignacio de Ulíbarri, J., González-Madroño, A., de Villar, N.G., et al. (2005) CONUT: A Tool for Controlling Nutritional Status. First Validation in a Hospital Population. Nutricion Hospitalaria, 20, 38-45.

[14] Shimada, M., Iwase, H., Hirashima, N., et al. (2017) Nutritional Status and Long-Term Prognosis in Patients with Refractory Hepatic Ascites Treated with Tolvaptan. Gastroenterol Hepatol Endosc, 2, 1-5.

https://doi.org/10.15761/GHE.1000131 